\title{
Rotary polygons in configurations
}

\author{
Marko Boben \\ Faculty of Computer Science, University of Ljubljana \\ Tržaška 25, 1000 Ljubljana, Slovenia \\ marko.boben@fri.uni-lj.si \\ Štefko Miklavič \\ University of Primorska \\ Primorska Institute of Natural Science and Technology \\ Muzejski trg 2, 6000 Koper, Slovenia \\ stefko.miklavic@upr.si \\ Primož Potočnik
}

Faculty of Mathematics and Physics, University of Ljubljana, and

Institute of Mathematics, Physics, and Mechanics

Jadranska 19, 1000 Ljubljana, Slovenia

primoz.potocnik@fmf.uni-lj.si

Submitted: Jun 4, 2010; Accepted: May 10, 2011; Published: May 23, 2011

Mathematics Subject Classifications: 05B30

\begin{abstract}
A polygon $A$ in a configuration $\mathcal{C}$ is called rotary if $\mathcal{C}$ admits an automorphism which acts upon $A$ as a one-step rotation. We study rotary polygons and their orbits under the group of automorphisms (and antimorphisms) of $\mathcal{C}$. We determine the number of such orbits for several symmetry types of rotary polygons in the case when $\mathcal{C}$ is flag-transitive. As an example, we provide tables of flag-transitive $\left(v_{3}\right)$ and $\left(v_{4}\right)$ configurations of small order containing information on the number and symmetry types of corresponding rotary polygons.
\end{abstract}

\section{Introduction}

Various problems regarding the polygons (or multilaterals) in configurations have been studied in the past. Even the earliest papers on configurations considered the existence of Hamiltonian polygons (see for example [11]) and the possibility of the decomposition of the configuration into polygons (see for example [10]). Another topic which attracted 


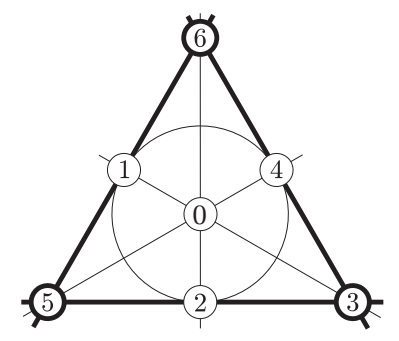

Figure 1: Strongly rotary 3-gon of the Fano plane (shown with thick points and lines).

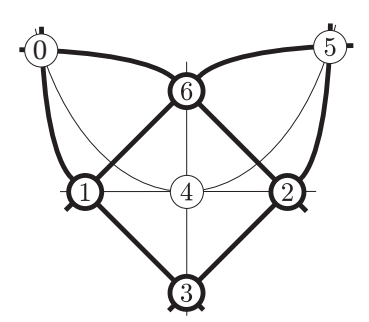

Figure 2: Strongly rotary 4-gon of the Fano plane.

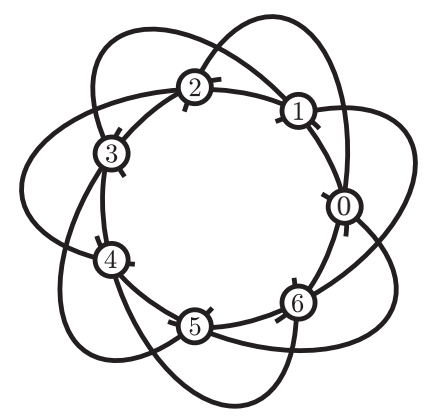

Figure 3: Rotary 7-gon of the Fano plane.

a considerable amount of attention is the existence or non-existence of $n$-gons in configurations; see [2] for more details on the history of this problem.

In this paper we focus on the rotary polygons (a notion that will be formally defined in Section 2) in flag-transitive combinatorial configurations. Before we start with precise definitions, let us take a look at the following example.

Consider the three drawings of the Fano plane $\mathcal{C}_{\mathcal{F}}$ in Figures 1-3, each emphasizing a particular polygon (denoted by thick lines and points). For each of these polygons there exists an automorphism of $\mathcal{C}_{\mathcal{F}}$ which rotates the polygon as follows:

$$
\left(\begin{array}{llll}
1 & 2 & 4
\end{array}\right)\left(\begin{array}{lll}
3 & 6 & 5
\end{array}\right), \quad\left(\begin{array}{llllll}
0 & 5
\end{array}\right)\left(\begin{array}{llllllll}
1 & 3 & 2 & 6
\end{array}\right), \quad\left(\begin{array}{lllllll}
0 & 1 & 2 & 3 & 4 & 5 & 6
\end{array}\right) .
$$

We call the polygon exhibiting such a symmetry to be rotary. The first two polygons are essentially different from the third one: For each of the first two polygons there exists an antimorphism of $\mathcal{C}_{\mathcal{F}}$ which acts on the $n$-gon - if viewed as an ordered sequence of points and lines - as a "rotation" of order $2 n$ :

$$
\begin{aligned}
& \left(\begin{array}{ll}
0 & 124
\end{array}\right)\left(\begin{array}{lllllll}
1 & 045 & 2 & 013 & 4 & 026
\end{array}\right)\left(\begin{array}{llllll}
3 & 346 & 6 & 156 & 5 & 235
\end{array}\right) \text {, } \\
& \left(\begin{array}{llll}
0 & 346 & 5 & 124
\end{array}\right)\left(\begin{array}{llllllll}
1 & 013 & 3 & 235 & 2 & 026 & 6 & 156
\end{array}\right)\left(\begin{array}{ll}
4 & 045
\end{array}\right)
\end{aligned}
$$

We will call such polygons strongly rotary.

On the other hand, there is no such antimorphism for the polygon in Figure 3, hence we call it weakly rotary.

Furthermore, the first two polygons admit a reflection in the group of automorphisms of $\mathcal{C}_{\mathcal{F}}$,

$$
\left(\begin{array}{ll}
1 & 4
\end{array}\right)\left(\begin{array}{ll}
3 & 5
\end{array}\right), \quad\left(\begin{array}{ll}
0 & 5
\end{array}\right)\left(\begin{array}{ll}
1 & 2
\end{array}\right)
$$




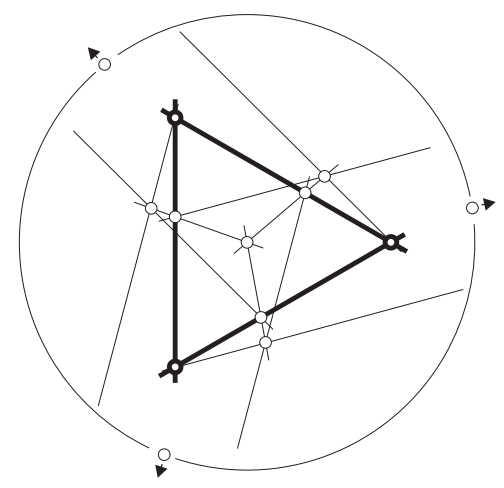

Figure 4: The flag-transitive $\left(13_{3}\right)$ configuration, and its chiral strongly rotary polygon (the triangle depicted by thick lines). Note that the configuration is realized with points and lines in the projective plane. The arrows indicate that the corresponding points are at infinity, together with the line through them.

while the third does not. However, there is an antimorphism

$$
\left(\begin{array}{ll}
0 & 013
\end{array}\right)\left(\begin{array}{ll}
1 & 026
\end{array}\right)(2 \quad 156)(3 \quad 045)\left(\begin{array}{lll}
4 & 346
\end{array}\right)\left(\begin{array}{lll}
5 & 235
\end{array}\right)\left(\begin{array}{ll}
6 & 124
\end{array}\right)
$$

of $\mathcal{C}_{\mathcal{F}}$ which reflects the third polygon (in a sense to be made clear in the next section). For this reason, all these polygons are called reflexive, the first two genuinely reflexive, and the third virtually reflexive.

Now consider the triangle in the $\left(13_{3}\right)$ configuration depicted in Figure 4 . It is clearly rotary, but it admits no reflection (neither an automorphism nor an antimorphism). We will call such polygons chiral.

In this paper we study rotary polygons and their orbits under the group of automorphisms (and antimorphisms) of the configuration. If the configuration is flag-transitive, we determine the number of such orbits. We conclude the paper with a series of illuminating examples and tables of flag-transitive $\left(v_{3}\right)$ and $\left(v_{4}\right)$ configurations of small order.

\section{Preliminaries}

A (combinatorial) configuration of type $\left(v_{r}, b_{k}\right)$ is an ordered triple $\mathcal{C}=(\mathcal{P}, \mathcal{L}, \mathcal{F})$ of mutually disjoint sets $\mathcal{P}, \mathcal{L}$ and $\mathcal{F} \subseteq\{\{p, \ell\}: p \in \mathcal{P}, \ell \in \mathcal{L}\}$ (whose elements are called, respectively, points, lines and flags) with $|\mathcal{P}|=v$ and $|\mathcal{L}|=b$ satisfying the following axioms:

(1) each line is incident with $k$ points;

(2) each point is incident with $r$ lines;

(3) two distinct points are incident with at most one common line; 
where a point $p$ is incident with a line $\ell$ if $\{p, \ell\} \in \mathcal{F}$.

A configuration is connected if for any two points $p$ and $q$ there exists a sequence $\left(p_{0}, \ell_{0}, p_{1}, \ell_{1}, \ldots, p_{n-1}, \ell_{n-1}, p_{n}\right)$ of points $p_{i}$ and lines $\ell_{i}$ such that $p_{0}=p$ and $p_{n}=q$ and $\ell_{i}$ is incident with $p_{i}$ and $p_{i+1}$ for each $0 \leq i<n$. All configurations considered in this paper are assumed to be connected.

If $\mathcal{C}=(\mathcal{P}, \mathcal{L}, \mathcal{F})$ is a configuration of type $\left(v_{r}, b_{k}\right)$, then $\mathcal{C}^{*}=(\mathcal{L}, \mathcal{P}, \mathcal{F})$ is a configuration of type $\left(b_{k}, v_{r}\right)$, called the dual configuration of $\mathcal{C}$.

An automorphism of a configuration $\mathcal{C}$ is an incidence-preserving permutation on the union $\mathcal{P} \cup \mathcal{L}$ which preserves each of the sets $\mathcal{P}$ and $\mathcal{L}$. Similarly, an antimorphism of a configuration $\mathcal{C}$ is an incidence-preserving permutation on $\mathcal{P} \cup \mathcal{L}$ which interchanges $\mathcal{P}$ and $\mathcal{L}$. The configuration $\mathcal{C}$ is said to be self-dual if it admits an antimorphism, that is, if it is isomorphic to its dual $\mathcal{C}^{*}$. Note that if $\mathcal{C}$ is self-dual, then $b=v$ and $k=r$. Whenever the latter happens, we say that $\mathcal{C}$ is symmetric of type $\left(v_{r}\right)$.

Following [8] we let $\operatorname{Aut}_{0}(\mathcal{C})$ denote the group of all automorphisms of $\mathcal{C}$, and we let Aut $(\mathcal{C})$ denote the group of all automorphisms and antimorphisms of $\mathcal{C}$ which happens to be the full automorphism group of the incidence graph of $\mathcal{C}$ (see Section 4 for the definition of incidence graph). Note that $\operatorname{Aut}_{0}(\mathcal{C})$ is a subgroup of $\operatorname{Aut}(\mathcal{C})$ of index at most 2.

We say that a configuration $\mathcal{C}=(\mathcal{P}, \mathcal{L}, \mathcal{F})$ is point-, line-, and flag-transitive if $\operatorname{Aut}(\mathcal{C})$ acts transitively on the sets $\mathcal{P}, \mathcal{L}, \mathcal{F}$, respectively. Moreover, a flag-transitive configuration $\mathcal{C}$ is strongly flag-transitive if $\operatorname{Aut}_{0}(\mathcal{C})$ acts transitively on $\mathcal{F}$, and is weakly flag-transitive otherwise. Note that a weakly flag-transitive configuration is necessarily self-dual.

A directed polygon (or more precisely, a directed $n$-gon) in a configuration is a cyclically ordered set $\left\{p_{0}, \ell_{0}, p_{1}, \ell_{1}, \ldots, \ell_{n-2}, p_{n-1}, \ell_{n-1}\right\}$ of pairwise distinct points $p_{i}$ and pairwise distinct lines $\ell_{i}$ such that $p_{i}$ is incident to $\ell_{i-1}$ and $\ell_{i}$ for each $i \in \mathbb{Z}_{n}$.

A directed $n$-gon $A=\left\{p_{0}, \ell_{0}, p_{1}, \ell_{1}, \ldots, \ell_{n-2}, p_{n-1}, \ell_{n-1}\right\}$ in $\mathcal{C}$ is said to be rotary if there exists $g \in \operatorname{Aut}(\mathcal{C})$ such that $p_{i}^{g}=p_{i+1}$ (and thus also $\ell_{i}^{g}=\ell_{i+1}$ ) for every $i \in \mathbb{Z}_{n}$. The above element $g$ is then called a shunt for $A$, and is necessarily an automorphism of $\mathcal{C}$. Similarly, $A$ is strongly rotary if there exists $g \in \operatorname{Aut}(\mathcal{C})$ such that $p_{i}^{g}=\ell_{i}$ (and $\left.\ell_{i}^{g}=p_{i+1}\right)$ for every $i \in \mathbb{Z}_{n}$. The element $g$ is then called a strong shunt for $A$, and is necessarily an antimorphism of $\mathcal{C}$. Directed polygons that are rotary but not strongly rotary will be called weakly rotary. Of course, strongly rotary polygons only exist in self-dual configurations.

Let $\mathcal{A}, \mathcal{A}_{s}$ and $\mathcal{A}_{w}$ denote the sets of all rotary, all strongly rotary, and all weakly rotary directed polygons, respectively. Note that each of the groups $\operatorname{Aut}(\mathcal{C})$ and $\operatorname{Aut}_{0}(\mathcal{C})$ acts naturally on the sets $\mathcal{A}$ and $\mathcal{A}_{s}$. For a group $G$ acting on a set $X$ we shall use the symbol $X / G$ to denote the set of all orbits of $G$ on $X$. In particular, the symbols $\mathcal{A} / G$, $\mathcal{A}_{s} / G$ and $\mathcal{A}_{w} / G$ will denote the sets of $G$-orbits of directed rotary, strongly rotary and weakly rotary polygons, respectively.

\section{Auxiliary results}

Throughout this section let $\mathcal{C}$ be a configuration of type $\left(v_{r}, b_{k}\right), G=\operatorname{Aut}(\mathcal{C})$ and $G_{0}=$ $\operatorname{Aut}_{0}(\mathcal{C})$. 
Lemma 3.1. With the notation above, the following hold:

(i) $\mathcal{A}_{s} / G_{0}=\mathcal{A}_{s} / G$

(ii) If $\mathcal{C}$ is self-dual then each $G$-orbit on $\mathcal{A}_{w}$ splits into two $G_{0}$-orbits (thus, $\left|\mathcal{A}_{w} / G_{0}\right|=$ $\left.2\left|\mathcal{A}_{w} / G\right|\right)$ and $\mathcal{A}_{w} / G_{0}=\mathcal{A}_{w} / G$ if $\mathcal{C}$ is not self-dual.

Proof. Let $A_{1}, A_{2} \in \mathcal{A}_{s}$ be in the same $G$-orbit, that is, $A_{2}=A_{1}^{h}$ for some $h \in G$, and let $g \in G$ be a strong shunt for $A_{2}$. Then either $h$ or $h g$ belongs to $G_{0}$. This shows that $A_{1}$ and $A_{2}$ are also in the same $G_{0}$-orbit, proving (i).

If $\mathcal{C}$ is not self-dual, then $G=G_{0}$ and (ii) clearly holds. Hence we may assume that the configuration is self-dual, and so $G_{0}$ is a subgroup of index $2 \mathrm{in} G$. In this case each $G$ orbit splits into at most two $G_{0}$-orbits, implying that $\left|\mathcal{A}_{w} / G_{0}\right| \leq 2\left|\mathcal{A}_{w} / G\right|$. What remains to show is that indeed every $G$-orbit of weakly rotary directed polygons contains two distinct $G_{0}$-orbits. Take $A \in \mathcal{A}_{w}$ and $h \in G \backslash G_{0}$. If $A$ and $A^{h}$ are in the same $G_{0}$ orbit, then there exists $h^{\prime} \in G_{0}$ such that $A^{h}=A^{h^{\prime}}$, and so $A^{h^{\prime} h^{-1}}=A$. By multiplying $h^{\prime} h^{-1}$ with an appropriate power $g^{n}$ of a shunt $g \in G_{0}$ of $A$, we obtain a strong shunt $h^{\prime} h^{-1} g^{n}$ of $A$, contradicting the fact that $A$ is weakly rotary. This implies that each $G$-orbit on $\mathcal{A}_{w}$ splits into two $G_{0}$-orbits.

Corollary 3.2. With the notation above, and assuming that $\mathcal{C}$ is self-dual, the following holds:

$$
\left|\mathcal{A} / G_{0}\right|=2|\mathcal{A} / G|-\left|\mathcal{A}_{s} / G\right|
$$

Proof. By Lemma 3.1 we see that

$$
\begin{aligned}
& 2|\mathcal{A} / G|-\left|\mathcal{A}_{s} / G\right|=2\left(\left|\mathcal{A}_{w} / G\right|+\left|\mathcal{A}_{s} / G\right|\right)-\left|\mathcal{A}_{s} / G\right|= \\
& =2\left|\mathcal{A}_{w} / G\right|+\left|\mathcal{A}_{s} / G\right|=\left|\mathcal{A}_{w} / G_{0}\right|+\left|\mathcal{A}_{s} / G_{0}\right|=\left|\mathcal{A} / G_{0}\right| .
\end{aligned}
$$

\section{Enumerating the orbits of rotary directed polygons}

Let $\mathcal{C}=(\mathcal{P}, \mathcal{L}, \mathcal{F})$ be a configuration of type $\left(v_{r}, b_{k}\right)$. Then $\mathcal{C}$ fully determines its incidence graph $\Gamma(\mathcal{C})$ (also called the Levi graph), whose vertex-set is $\mathcal{P} \cup \mathcal{L}$, with $p \in \mathcal{P}$ adjacent to $\ell \in \mathcal{L}$ whenever $p$ is incident with $\ell$. Note that $\Gamma(\mathcal{C})$ is a bi-regular bipartite graph of valence $(k, r)$ and girth at least 6 . (A bipartite graph is called bi-regular if the vertices of the same bipartition set have the same valence.) Conversely, each bi-regular bipartite graph with girth at least 6 determines a pair of mutually dual configurations, whose points are vertices in one bipartition set, lines are vertices in the other bipartition set, and incidence relation is the adjacency relation in $\Gamma$. Note that a configuration is connected if and only if its Levi graph is connected.

Clearly $\operatorname{Aut}(\mathcal{C})=\operatorname{Aut}(\Gamma(\mathcal{C}))$, where the subgroup $\operatorname{Aut}_{0}(\mathcal{C})$ coincides with the group $\operatorname{Aut}_{0}(\Gamma(\mathcal{C}))$ preserving each set of the bipartition. The notions of weak and strong flagtransitivity translate into the language of group actions on graphs as follows. For the 
graph-theoretical notions not defined here, as well as the proof of the theorem below, we refer the reader to [8].

Proposition 4.1. Let $\mathcal{C}$ be a configuration and let $\Gamma$ be its incidence graph. Let $G=$ $\operatorname{Aut}(\mathcal{C})=\operatorname{Aut}(\Gamma)$, and let $G_{0}=\operatorname{Aut}_{0}(\mathcal{C})=\operatorname{Aut}_{0}(\Gamma)$ be the group of automorphisms of $\mathcal{C}$, also viewed as the bipartition preserving subgroup of $\operatorname{Aut}(\Gamma)$. Then

(i) $\mathcal{C}$ is strongly flag-transitive if and only if $G_{0}$ acts locally arc-transitively on $\Gamma$ (that is, if and only if the stabilizer in $G_{0}$ of any vertex $v$ of $\Gamma$ acts transitively on the neighbourhood of $v$ ).

(ii) $\mathcal{C}$ is strongly flag-transitive and self-dual if and only if $G$ acts arc-transitively on $\Gamma$.

(iii) $\mathcal{C}$ is weakly flag-transitive if and only if $G$ acts $\frac{1}{2}$-arc-transitively on $\Gamma$.

Note that a directed $n$-gon $A$ in $\mathcal{C}$ can be viewed as a directed cycle $C_{A}$ of length $2 n$ in $\Gamma(\mathcal{C})$. If $A$ is strongly rotary, then a strong shunt of $A$ corresponds to an automorphism of $\Gamma$ preserving and rotating $C_{A}$ one step forward. Cycles of this type were first studied by Conway (see [1]), where they were called consistent cycles. Similarly, if $A$ is rotary, then a shunt of $A$ corresponds to a two-step rotation of $C_{A}$. To distinguish between these two types of cycles, the directed cycles admitting a 2 -step rotation will be called $\frac{1}{2}$-consistent. More generally, if $\Gamma$ is a graph and $G \leq \operatorname{Aut}(\Gamma)$, then a directed cycle $C$ for which there exists $g \in G$ acting as a $k$-step rotation on $C$ is called $\left(G, \frac{1}{k}\right)$-consistent.

The following result about consistent cycles in edge-transitive graphs was proved in [9] (parts (i) and (ii) of the theorem below) and [3] (part (iii)).

Theorem 4.2. [3, 9] Let $\Gamma$ be a bi-regular graph of valence $\left(d, d^{\prime}\right)$ and let $G$ be an edgetransitive subgroup of $\operatorname{Aut}(\Gamma)$. Then the following hold:

(i) If $G$ acts transitively on the arcs of $\Gamma$, then $d=d^{\prime}$ and there are precisely $(d-1)$ $G$-orbits of $(G, 1)$-consistent directed cycles and precisely $\frac{d(d-1)}{2} G$-orbits of $\left(G, \frac{1}{2}\right)$ consistent directed cycles in $\Gamma$.

(ii) If $G$ acts locally arc-transitively but not arc-transitively on $\Gamma$, then there are no $(G, 1)$-consistent directed cycles and precisely $(d-1)\left(d^{\prime}-1\right)$ G-orbits of $\left(G, \frac{1}{2}\right)$ consistent directed cycles in $\Gamma$.

(iii) If $G$ acts $\frac{1}{2}$-arc-transitively on $\Gamma$, then $d=d^{\prime}$ and there are precisely $d$-orbits of $(G, 1)$-consistent directed cycles and precisely $\frac{d^{2}-d+2}{2} G$-orbits of $\left(G, \frac{1}{2}\right)$-consistent directed cycles in $\Gamma$.

The above theorem yields the following result about orbits of rotary directed polygons in configurations.

Theorem 4.3. Let $\mathcal{C}$ be a configuration of type $\left(v_{r}, b_{k}\right)$, let $G=\operatorname{Aut}(\mathcal{C})$ and let $G_{0}=$ $\operatorname{Aut}_{0}(\mathcal{C})$.

If $\mathcal{C}$ is strongly flag-transitive and non-self-dual, then 
(i) $|\mathcal{A} / G|=\left|\mathcal{A} / G_{0}\right|=(k-1)(r-1)$;

(ii) $\left|\mathcal{A}_{s} / G\right|=\left|\mathcal{A}_{s} / G_{0}\right|=0$.

If $\mathcal{C}$ is strongly flag-transitive and self-dual, then

(iii) $|\mathcal{A} / G|=\frac{r(r-1)}{2}$ and $\left|\mathcal{A} / G_{0}\right|=(r-1)^{2}$;

(iv) $\left|\mathcal{A}_{s} / G\right|=\left|\mathcal{A}_{s} / G_{0}\right|=r-1$.

If $\mathcal{C}$ is weakly flag-transitive (and thus self-dual), then

(v) $|\mathcal{A} / G|=\frac{r^{2}-r+2}{2}$ and $\left|\mathcal{A} / G_{0}\right|=r^{2}-2 r+2$;

(vi) $\left|\mathcal{A}_{s} / G\right|=\left|\mathcal{A}_{s} / G_{0}\right|=r$.

Remark 4.4. Since $\mathcal{A}$ is disjoint union of $\mathcal{A}_{s}$ and $\mathcal{A}_{w}$ then in both self-dual cases it follows from the equations that $\left|\mathcal{A}_{w} / G\right|=\frac{(r-1)(r-2)}{2}$ and $\left|\mathcal{A}_{w} / G_{0}\right|=(r-1)(r-2)$.

Proof. Recall that rotary directed polygons in $\mathcal{C}$ correspond to $\left(G, \frac{1}{2}\right)$-consistent directed cycles in the incidence graph $\Gamma=\Gamma(\mathcal{C})$ (which are then also $\left(G_{0}, \frac{1}{2}\right)$-consistent), while strongly rotary directed polygons in $\mathcal{C}$ correspond to $(G, 1)$-consistent directed cycles in $\Gamma$. Further, since the type of $\mathcal{C}$ is $\left(v_{r}, b_{k}\right)$, the graph $\Gamma$ is bi-regular of valence $\left(d, d^{\prime}\right)=(r, k)$.

Assume first that $\mathcal{C}$ is strongly flag-transitive.

If $\mathcal{C}$ is non-self-dual, then $G=G_{0}$ acts locally arc-transitively on $\Gamma$, and (i) follows directly from part (ii) of Theorem 4.2. Part (ii) is obvious, since there are no strongly rotary polygons in a non-self-dual configuration.

If $\mathcal{C}$ is self-dual, then $G$ acts arc-transitively on $\Gamma$, while $G_{0}$ acts locally arc-transitively but not arc-transitively. The first claim of part (iii) then follows directly from part (i) of Theorem 4.2. Part (iv) is a consequence of part (i) of Theorem 4.2 and part (i) of Lemma 3.1. The second claim of part (iii) now follows from Corollary 3.2.

Assume now that $\mathcal{C}$ is weakly flag-transitive. Then $\mathcal{C}$ is self-dual and $G$ acts $\frac{1}{2}$-arctransitively on $\Gamma$. The first claim of part (v) follows directly from part (iii) of Theorem 4.2, while part (vi) follows from part (iii) of Theorem 4.2 and part (i) of Lemma 3.1. Finally, the second claim of part (v) follows from Corollary 3.2.

\section{Reflexive and chiral undirected polygons}

Thus far we have only considered directed polygons, where there is a distinction between a directed polygon $A=\left\{p_{0}, \ell_{0}, \ldots, p_{n-1}, \ell_{n-1}\right\}$ and its inverse $A^{-1}=\left\{p_{0}, \ell_{n-1}, \ldots, p_{1}, \ell_{0}\right\}$. The inverse of a directed rotary polygon $A$ in $\mathcal{C}$ is clearly also rotary. If $A$ and $A^{-1}$ belong to the same orbit under $\operatorname{Aut}(\mathcal{C})$, then we say that $A$ is reflexive. There are two essentially distinct types of reflexive polygons. Namely, it may happen that $A$ can be mapped to $A^{-1}$ by an automorphism of $\mathcal{C}$; in this case, we shall say that $A$ is genuinely reflexive. On the other hand, if every $g \in \operatorname{Aut}(\mathcal{C})$ which maps $A$ to $A^{-1}$ is an antimorphism of $\mathcal{C}$, then 
we say that $A$ is virtually reflexive. A directed rotary polygon which is not reflexive is called chiral.

Note that every reflexive strongly rotary directed polygon is necessarily genuinely reflexive. Indeed, let $\tau \in \operatorname{Aut}(\mathcal{C})$ be a reflection of a strongly rotary directed polygon $A$ in a configuration $\mathcal{C}$, and let $g$ be its strong shunt. Then either $\tau$ or $g \tau$ is a reflection of $A$ contained in $\operatorname{Aut}_{0}(\mathcal{C})$. Hence $A$ is genuinely reflexive.

Furthermore, if $A$ is a reflexive directed polygon in a weakly flag-transitive configuration $\mathcal{C}$, then $A$ is genuinely reflexive and weakly rotary. Indeed, if $A$ is either strongly rotary or virtually reflexive, then there exists an antimorphism of $\mathcal{C}$ which acts as reflection on $A$. Combining this antimorphism by an appropriate rotation of $A$ (if necessary), we obtain an antimorphism of $\mathcal{C}$ preserving a flag of $\mathcal{C}$. But this is impossible if $\mathcal{C}$ is weakly flag-transitive.

Let us now turn our attention to (undirected) polygons, which may abstractly be thought of as pairs of mutually inverse directed polygons. We shall extend all the relevant notions defined for directed polygons in the natural way to their underlying polygons. For example, a polygon underlying a directed polygon $A$ is called rotary if $A$ is rotary.

Note that there is a one-to-one correspondence between the $\operatorname{Aut}(\mathcal{C})$-orbits of reflexive directed polygons and the $\operatorname{Aut}(\mathcal{C})$-orbits of reflexive undirected polygons, and that each $\operatorname{Aut}(\mathcal{C})$-orbit of chiral undirected polygons corresponds to two $\operatorname{Aut}(\mathcal{C})$-orbits of chiral directed polygons (one containing the inverses of the other). Similarly, there is a one-toone correspondence between the $\operatorname{Aut}_{0}(\mathcal{C})$-orbits of genuinely reflexive directed polygons and the $\operatorname{Aut}_{0}(\mathcal{C})$-orbits of genuinely reflexive undirected polygons. Also, each $\mathrm{Aut}_{0}(\mathcal{C})$ orbit of virtually reflexive or chiral polygons corresponds to two $\operatorname{Aut}_{0}(\mathcal{C})$-orbits of virtually reflexive or chiral directed polygons.

Let $s^{+}, s^{-}$and $c$ denote the number of $\operatorname{Aut}(\mathcal{C})$-orbits of genuinely reflexive, virtually reflexive, and chiral undirected polygons, respectively, and let $s_{0}^{+}, s_{0}^{-}$and $c_{0}$ denote the number of $\operatorname{Aut}_{0}(\mathcal{C})$-orbits of genuinely reflexive, virtually reflexive, and chiral undirected polygons, respectively. The following corollary now follows directly from the above comments and Theorem 4.3.

Corollary 5.1. Let $\mathcal{C}$ be a configuration of type $\left(v_{r}, b_{k}\right)$, and let $s^{+}, s^{-}, c, s_{0}^{+}, s_{0}^{-}, c_{0}$ be as above.

(i) If $\mathcal{C}$ is strongly flag-transitive and non-self-dual, then $s^{-}=s_{0}^{-}=0, s^{+}=s_{0}^{+}, c=c_{0}$, and $s^{+}+2 c=(k-1)(r-1)$.

(ii) If $\mathcal{C}$ is strongly flag-transitive and self-dual, then $s^{+}+s^{-}+2 c=\frac{r(r-1)}{2}$ and $s_{0}^{+}+$ $2 s_{0}^{-}+2 c_{0}=(r-1)^{2}$.

(iii) If $\mathcal{C}$ is weakly flag-transitive (and thus self-dual), then $s^{-}=s_{0}^{-}=0, s^{+}+2 c=\frac{r^{2}-r+2}{2}$ and $s_{0}^{+}+2 c_{0}=r^{2}-2 r+2$.

Finally, let us comment on the relationship between the orbits of directed and undirected polygons under the groups $\operatorname{Aut}(\mathcal{C})$ and $\operatorname{Aut}_{0}(\mathcal{C})$.

Let $A$ be a weakly rotary directed polygon. 
If $A$ is chiral, then its inverse $A^{-1}$ is in a different orbit, both under $\operatorname{Aut}(\mathcal{C})$ as well as under $\operatorname{Aut}_{0}(\mathcal{C})$. Moreover, by Lemma 3.1, the $\operatorname{Aut}(\mathcal{C})$-orbit of $A$ splits into two chiral $\operatorname{Aut}_{0}(\mathcal{C})$-orbits (let us denote the two representatives by $A_{1}$ and $A_{2}$ ). Hence there are four distinct $\operatorname{Aut}_{0}(\mathcal{C})$-orbits associated with $A$, the representatives of which are $A_{1}, A_{1}^{-1}, A_{2}$ and $A_{2}^{-1}$. These four orbits thus give rise to two $\operatorname{Aut}_{0}(\mathcal{C})$-orbits (as well as $\operatorname{Aut}(\mathcal{C})$-orbits) of undirected polygons.

If $A$ is virtually reflexive, then the $\operatorname{Aut}(\mathcal{C})$-orbit of $A$ splits into two $\operatorname{Aut}_{0}(\mathcal{C})$-orbits, one containing $A$ and the other containing $A^{-1}$. Hence there is a unique $\operatorname{Aut}_{0}(\mathcal{C})$-orbit of undirected polygons associated with $A$.

Finally, if $A$ is genuinely reflexive, then the $\operatorname{Aut}(\mathcal{C})$-orbit of $A$ splits into two $\operatorname{Aut}_{0}(\mathcal{C})$ orbits, each of which is closed under taking inverses of the polygons. This implies that there exist two $\operatorname{Aut}_{0}(\mathcal{C})$-orbits of undirected polygons associated with $A$ which merge into a single orbit under $\operatorname{Aut}(\mathcal{C})$.

\section{$6 \quad$ Examples}

In this section, we present several examples demonstrating the theory developed in the previous sections. In particular, we concentrate on the flag-transitive $\left(v_{3}\right)$ and $\left(v_{4}\right)$ configurations. Note that each of these configurations belongs to exactly one of the following classes:

- self-dual strongly flag-transitive $\left(v_{3}\right)$ configurations;

- non-self-dual strongly flag-transitive $\left(v_{3}\right)$ and $\left(v_{4}\right)$ configurations;

- self-dual strongly flag-transitive $\left(v_{4}\right)$ configurations;

- weakly flag-transitive (and thus self-dual) $\left(v_{4}\right)$ configurations.

For each of these classes we provide a list of its members of small orders. These lists were extracted from the following sources:

- the census of cubic arc-transitive graphs [4] for self-dual strongly flag-transitive $\left(v_{3}\right)$ configurations;

- the census of cubic semisymmetric graphs [6] for non-self-dual strongly flag-transitive $\left(v_{3}\right)$ configurations;

- the database of tetravalent edge-transitive graphs [12] for the three types of flagtransitive $\left(v_{4}\right)$ configurations.

Note that the tables of $\left(v_{3}\right)$ configurations are complete up to the order of the largest member in the list, however, the completeness of lists of $\left(v_{4}\right)$ configurations can not be guaranteed.

The lists are organized in tables, collected in Section 7 at the end of the paper, where each line corresponds to one configuration. The first column in each line contains the 
information on the order of the configuration, and the other columns contain the information on the length of polygons and the symmetry type of the $\operatorname{Aut}(\mathcal{C})$-orbits of the directed rotary polygons. Each $\operatorname{Aut}(\mathcal{C})$-orbit is represented by a symbol of the form $n X$, where $n$ denotes the length of the polygon in the orbit and $X \in\left\{S^{+}, S^{-}, C\right\}$ denotes the symmetry type of the polygon (where $S^{+}, S^{-}$, and $C$ stand for genuinely reflexive, virtually reflexive, and chiral, respectively).

\subsection{Self-dual strongly flag-transitive $\left(v_{3}\right)$ configurations}

Plugging $r=3$ into Theorem 4.3 (iii) and (iv), a self-dual strongly flag-transitive $\left(v_{3}\right)$ configuration $\mathcal{C}$ has precisely three $\operatorname{Aut}(\mathcal{C})$-orbits of directed rotary polygons. Precisely one of these orbits consists of weakly rotary polygons. Note that since chiral orbits come in pairs this orbit of weakly rotary polygons must be reflexive (genuinely or virtually). The other two orbits consist of strongly rotary polygons, which may therefore all be either genuinely reflexive or chiral. We may encode the above possibilities by the symbols $\left(S^{+} S^{+} \mid S^{+}\right),\left(S^{+} S^{+} \mid S^{-}\right),\left(C C \mid S^{+}\right)$and $\left(C C \mid S^{-}\right)$, respectively. For example, the symbol $\left(S^{+} S^{+} \mid S^{-}\right)$corresponds to the situation where the two strongly rotary orbits are genuinely reflexive and the weakly rotary orbit is virtually reflexive. All four possibilities indeed occur. The smallest configurations of given types are: the Fano plane on 7 points for type $\left(S^{+} S^{+} \mid S^{-}\right)$, the Pappus configuration on 9 points for type $\left(S^{+} S^{+} \mid S^{+}\right)$, the $\left(13_{3}\right)$ configuration for type $\left(C C \mid S^{-}\right)$(its incidence graph is the unique connected arc-transitive cubic graph on 26 points and can be found in the Foster census under name F26A), and the $\left(224_{3}\right)$ configuration for type $\left(C C \mid S^{+}\right)$. It is worth noting that the incidence graph of the latter is the smallest cubic arc-transitive graph of girth 14, implying in particular that the configuration itself contains no $k$-gons for $k \leq 6$.

Recall that by Lemma 3.1 the two strongly rotary $\operatorname{Aut}(\mathcal{C})$-orbits coincide with the two strongly rotary $\mathrm{Aut}_{0}(\mathcal{C})$-orbits, while the weakly rotary $\operatorname{Aut}(\mathcal{C})$-orbit splits into two $\operatorname{Aut}_{0}(\mathcal{C})$-orbits, giving four $\operatorname{Aut}_{0}(\mathcal{C})$-orbits of directed rotary polygons in total.

Finally, it follows from the above comments that there are either two or three $\operatorname{Aut}(\mathcal{C})$ orbits of undirected rotary polygons, two if $\mathcal{C}$ is of type $\left(C C \mid S^{+}\right)$or $\left(C C \mid S^{-}\right)$and three if $\mathcal{C}$ is of type $\left(S^{+} S^{+} \mid S^{+}\right)$or $\left(S^{+} S^{+} \mid S^{-}\right)$. Similarly, there are two, three or four orbits of undirected rotary polygons under the group $\operatorname{Aut}_{0}(\mathcal{C})$; two if $\mathcal{C}$ is of type $\left(C C \mid S^{-}\right)$, three if $\mathcal{C}$ is of type $\left(C C \mid S^{+}\right)$or $\left(S^{+} S^{+} \mid S^{-}\right)$, and four if $\mathcal{C}$ is of type $\left(S^{+} S^{+} \mid S^{+}\right)$.

The list of all self-dual strongly flag-transitive $\left(v_{3}\right)$ configurations on up to 63 points is given in Table 1.

Several well-known configurations can be found in Table 1. Let us have a closer look at some of them.

In the introduction we have already considered the Fano plane (Figures 1, 2, 3) of type $\left(S^{+} S^{+} \mid S^{-}\right)$; that is, with two Aut $(\mathcal{C})$-orbits of genuinely reflexive strongly rotary directed polygons and one $\operatorname{Aut}(\mathcal{C})$-orbit of virtually reflexive weakly rotary directed polygons.

Another well-known strongly flag-transitive configuration is the Pappus $\left(9_{3}\right)$ configuration, shown in Figure 5, illustrating the Pappus theorem. Its symmetry type is $\left(S^{+} S^{+} \mid S^{+}\right)$, giving rise to three orbits of undirected rotary polygons under the group 


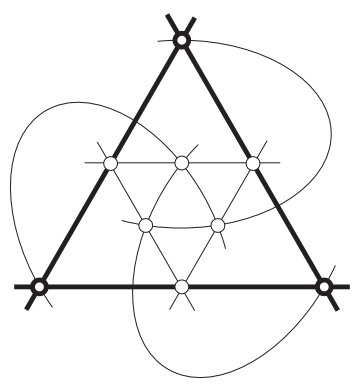

(a)

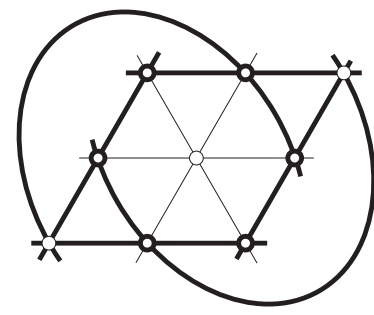

(c)

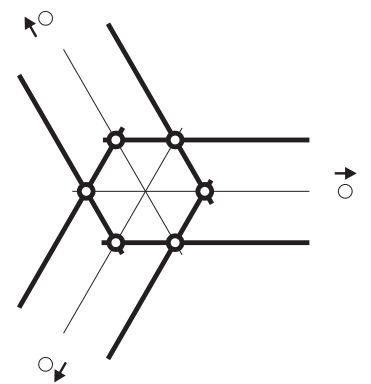

(b)

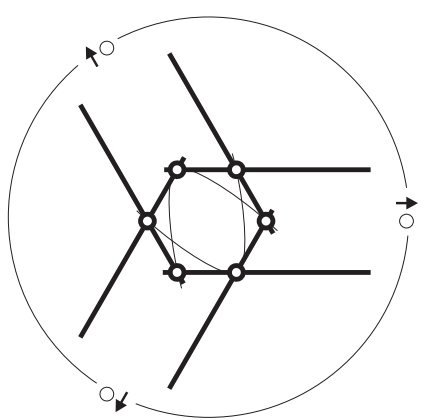

(d)

Figure 5: Rotary polygons in the Pappus configuration shown with thick points and lines. Polygons (a) and (b) are strongly rotary while (c) and (d) are weakly rotary.

Aut $(\mathcal{C})$. The representatives of the two orbits of strongly rotary polygons are shown in figures (a) and (b), while figures (c) and (d) show two weakly rotary polygons, which belong to the same $\operatorname{Aut}(\mathcal{C})$-orbit but to distinct $\operatorname{Aut}_{0}(\mathcal{C})$-orbits. Note that in $(\mathrm{b})$ we are able to realize the configuration with points and lines in the projective plane (arrows indicate that the corresponding points are at infinity) and simultaneously showing the rotary polygon as a regular hexagon.

The Desargues $\left(10_{3}\right)$ configuration, associated with the well-known Desargues theorem, is also strongly flag-transitive. Thus the number of rotary polygons can be determined from Theorem 4.3. We show them in Figure 6. Here again we can realize the configuration with points and lines in the (projective) plane and showing the rotary polygons as regular polygons where possible. Here, also, all rotary polygons are genuinely reflexive, thus giving rise to four $\mathrm{Aut}_{0}(\mathcal{C})$-orbits of undirected rotary polygons. The two $\mathrm{Aut}_{0}(\mathcal{C})$-orbits of weakly rotary polygons are shown as (c) and (d).

Among other flag-transitive $\left(v_{3}\right)$ configurations listed in Table 1, let us mention the Cremona-Richmond $\left(15_{3}\right)$ configuration which is the smallest triangle-free $\left(v_{3}\right)$ configuration, see [2]. 


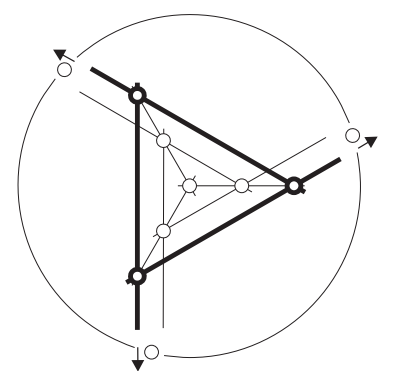

(a)

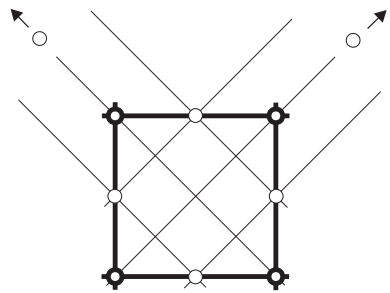

(c)

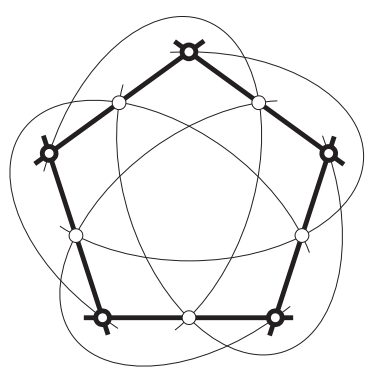

(b)

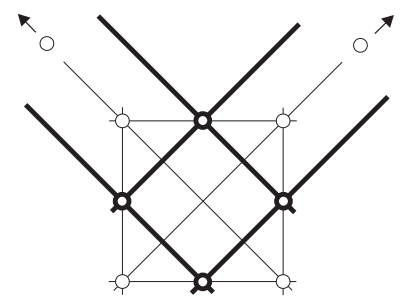

(d)

Figure 6: Rotary polygons in the Desargues configuration shown with thick points and lines. Polygons (a) and (b) are strongly rotary while (c) and (d) are weakly rotary.

\subsection{Non-self-dual strongly flag-transitive $\left(v_{r}\right)$ configurations}

Since a non-self-dual flag-transitive configuration permits no antimorphisms, every rotary polygon is necessarily weakly-rotary, and every reflexive rotary polygon in necessarily genuinely reflexive. Moreover, every such configuration is in fact strongly flag-transitive.

By Theorem 4.3 (i), a non-self-dual strongly flag-transitive $\left(v_{r}\right)$ configuration $\mathcal{C}$ has precisely $(r-1)^{2}$ orbits of directed rotary polygons under the group $\operatorname{Aut}_{0}(\mathcal{C})=\operatorname{Aut}(\mathcal{C})$. Since orbits of chiral directed polygons come in pairs, there exists at least one genuinely reflexive orbit whenever $r$ is even. Note that when $r=3$, exactly three possible combinations for the symmetry types of rotary polygons exist: all four orbits are genuinely reflexive, type $\left(S^{+} S^{+} S^{+} S^{+}\right)$, all four orbits are chiral, type $(C C C C)$, or two orbits are genuinely reflexive and two are chiral, type $\left(C C S^{+} S^{+}\right)$. The data in Table 2 shows that all three possibilities in fact occur. Similarly, when $r=4$, there are five possible combinations for the symmetry types with one, three, five, seven or nine genuinely reflexive orbits, respectively. As one may deduce from Table 3, all these types occur.

The smallest configuration in Table 2 on 27 points arises from the well-known Gray graph. A drawing of the third smallest strongly flag-transitive non-self-dual $\left(v_{3}\right)$ configuration can be found in [5].

\subsection{Self-dual strongly flag-transitive $\left(v_{4}\right)$ configurations}

By Theorem 4.3 (iii) and (iv), a self-dual strongly flag-transitive $\left(v_{4}\right)$ configuration $\mathcal{C}$ has precisely six $\operatorname{Aut}(\mathcal{C})$-orbits of directed rotary polygons, out of which precisely three 


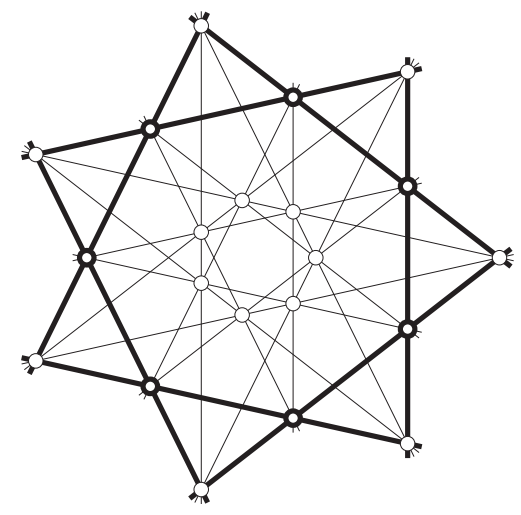

(a)

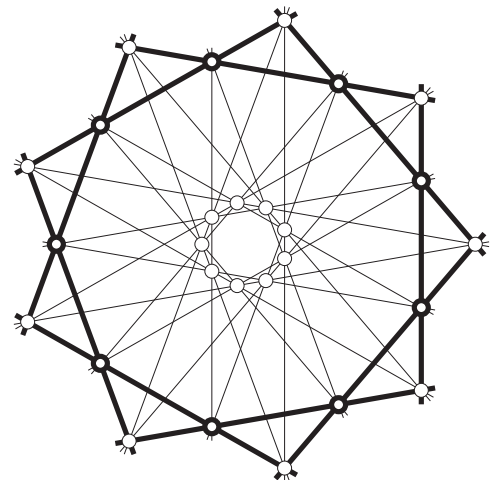

(b)

Figure 7: Klein $\left(21_{4}\right)$ configuration with indicated strongly rotary genuinely reflexive polygon of length 7 (a), and the smallest known weakly flag-transitive configuration (b).

consist of strongly rotary polygons. This implies that there exists at least one genuinely reflexive orbit of strongly rotary polygons and at least one reflexive (genuinely or virtually) of weakly rotary polygons. Hence there are two possible symmetry types for the three Aut $(\mathcal{C})$-orbits of strongly rotary polygons: $\left(S^{+} S^{+} S^{+}\right)$and $\left(C C S^{+}\right)$. Similarly, there are six possibilities for the symmetry types of the weakly rotary polygons: four types with all three orbits reflexive (each either genuinely or virtually), and two types with two chiral orbits and one reflexive (again either genuinely or virtually). This amounts to 12 possible symmetry types for the $\operatorname{six} \operatorname{Aut}(\mathcal{C})$-orbits of directed rotary polygons.

In Table 4 we provide a list of self-dual strongly flag-transitive $\left(v_{4}\right)$ configurations. The list is based on the census of tetravalent edge-transitive graphs available in [12]. Since this census may not be complete, Table 4 may be missing some configurations even in the range up to 48 points.

The computational data in Table 4 show that 5 of the possible 12 symmetry types indeed occur. It remains an open question whether there exists a self-dual strongly flagtransitive $\left(v_{4}\right)$ configurations of any the following 7 types: $\left(S^{+} S^{+} S^{+} \mid C C S^{+}\right),\left(S^{+} S^{+} S^{+} \mid\right.$ $\left.C C S^{-}\right),\left(C C S^{+} \mid S^{+} S^{+} S^{+}\right),\left(C C S^{+} \mid S^{-} S^{+} S^{+}\right),\left(C C S^{+} \mid S^{-} S^{-} S^{+}\right),\left(C C S^{+} \mid C C S^{+}\right)$, $\left(C C S^{+} \mid S^{-} S^{-} S^{-}\right)$.

We mention that the smallest configuration in Table 4 on 13 points arises from the projective plane of order 3. Among other strongly flag-transitive $\left(v_{4}\right)$ configurations, let us mention the Klein $\left(21_{4}\right)$ configuration studied by Grünbaum and Rigby [7], see Figure 7 (a).

\subsection{Weakly flag-transitive $\left(v_{4}\right)$ configurations}

In general, for weakly-flag transitive $\left(v_{4}\right)$ configurations, Theorem 4.3 (vi) says that there are four orbits of strongly rotary polygons and three orbits of weakly rotary polygons under $\operatorname{Aut}(\mathcal{C})$. Since $\operatorname{Aut}(\mathcal{C})$ acts $\frac{1}{2}$-arc-transitively on the Levi graph $\Gamma$, none of the orbits of strongly rotary polygons is reflexive, while one of the three orbits of weakly rotary 
polygons is genuinely reflexive and the other two are chiral, see [3, Section 6.1]. Hence all weakly flag-transitive $\left(v_{4}\right)$ configurations are of symmetry type $\left(C C C C \mid C C S^{+}\right)$.

This implies that there are precisely four $\operatorname{Aut}(\mathcal{C})$-orbits of undirected rotary polygons, two of them being strongly rotary and two weakly rotary. Moreover, in view of discussion beneath Corollary 5.1 it follows that each of the $\operatorname{Aut}(\mathcal{C})$-orbits of weakly rotary undirected polygons splits into two $\operatorname{Aut}_{0}(\mathcal{C})$-orbits, while each of the strongly rotary $\operatorname{Aut}(\mathcal{C})$-orbits is also an orbit under $\operatorname{Aut}_{0}(\mathcal{C})$. This gives us two $\operatorname{Aut}_{0}(\mathcal{C})$-orbits of strongly rotary undirected polygons (both being chiral) and four $\mathrm{Aut}_{0}(\mathcal{C})$-orbits of weakly rotary undirected polygons (two of them being genuinely reflexive and two chiral).

A (not necessarily complete) list of weakly flag-transitive $\left(v_{4}\right)$ configurations on up to 63 points can be found in Table 5 .

An example of a weakly-flag transitive $\left(v_{4}\right)$ configuration from [8], the smallest known such configuration, is shown in Figure 7 (b). The indicated 9-gon is genuinely reflexive and therefore weakly rotary. 


\section{$\begin{array}{ll}7 & \text { Tables }\end{array}$}

\begin{tabular}{||c||cc|c||c||cc|c||}
\hline$v$ & Strongly rotary & Weakly rotary & $v$ & \multicolumn{2}{|c||}{ Strongly rotary } & Weakly rotary \\
\hline 7 & $3 S^{+}$ & $4 S^{+}$ & $7 S^{-}$ & 39 & $3 C$ & $3 C$ & $39 S^{-}$ \\
8 & $4 S^{+}$ & $6 S^{+}$ & $8 S^{-}$ & 40 & $10 S^{+}$ & $12 S^{+}$ & $8 S^{+}$ \\
9 & $3 S^{+}$ & $6 S^{+}$ & $6 S^{+}$ & 43 & $3 C$ & $3 C$ & $43 S^{-}$ \\
10 & $3 S^{+}$ & $5 S^{+}$ & $4 S^{+}$ & 45 & $5 S^{+}$ & $12 S^{+}$ & $6 S^{+}$ \\
12 & $3 S^{+}$ & $6 S^{+}$ & $4 S^{-}$ & 48 & $3 S^{+}$ & $12 S^{+}$ & $8 S^{-}$ \\
13 & $3 C^{-}$ & $3 C$ & $13 S^{-}$ & 48 & $4 S^{+}$ & $6 S^{+}$ & $6 S^{+}$ \\
15 & $4 S^{+}$ & $5 S^{+}$ & $6 S^{+}$ & 49 & $3 C$ & $3 C$ & $49 S^{-}$ \\
16 & $3 S^{+}$ & $4 S^{+}$ & $8 S^{-}$ & 49 & $3 S^{+}$ & $7 S^{+}$ & $14 S^{-}$ \\
19 & $3 C$ & $3 C$ & $19 S^{-}$ & 52 & $3 C$ & $3 C$ & $26 S^{-}$ \\
20 & $5 S^{+}$ & $6 S^{+}$ & $4 S^{+}$ & 55 & $5 S^{+}$ & $6 S^{+}$ & $5 S^{+}$ \\
21 & $3 C$ & $3 C$ & $21 S^{-}$ & 56 & $6 C$ & $6 C$ & $28 S^{-}$ \\
24 & $6 S^{+}$ & $12 S^{+}$ & $8 S^{-}$ & 56 & $4 S^{+}$ & $7 S^{+}$ & $6 S^{-}$ \\
25 & $3 S^{+}$ & $5 S^{+}$ & $10 S^{-}$ & 56 & $4 S^{+}$ & $14 S^{+}$ & $8 S^{+}$ \\
27 & $3 S^{+}$ & $9 S^{+}$ & $6 S^{-}$ & 57 & $3 C$ & $3 C$ & $57 S^{-}$ \\
28 & $3 C$ & $3 C$ & $14 S^{-}$ & 60 & $4 S^{+}$ & $5 S^{+}$ & $5 S^{-}$ \\
28 & $4 S^{+}$ & $7 S^{+}$ & $8 S^{+}$ & 60 & $5 S^{+}$ & $15 S^{+}$ & $6 S^{-}$ \\
31 & $3 C$ & $3 C$ & $31 S^{-}$ & 61 & $3 C$ & $3 C$ & $61 S^{-}$ \\
32 & $4 S^{+}$ & $6 S^{+}$ & $8 S^{-}$ & 63 & $3 C$ & $3 C$ & $21 S^{-}$ \\
36 & $3 S^{+}$ & $6 S^{+}$ & $12 S^{-}$ & $\ldots$ & $\ldots$ & $\ldots$ & $\ldots$ \\
37 & $3 C$ & $3 C$ & $37 S^{-}$ & 224 & $7 C$ & $7 C$ & $8 S^{+}$ \\
\hline
\end{tabular}

Table 1: Self-dual strongly flag-transitive $\left(v_{3}\right)$ configurations, $v \leq 63$, together with the smallest example of type $\left(C C \mid S^{+}\right)$with $v=224$.

\begin{tabular}{||c||cccc||}
\hline$v$ & \multicolumn{4}{|c||}{ Weakly rotary orbits } \\
27 & $4 S^{+}$ & $6 S^{+}$ & $6 S^{+}$ & $9 S^{+}$ \\
55 & $5 S^{+}$ & $10 S^{+}$ & $11 S^{+}$ & $12 S^{+}$ \\
56 & $6 C$ & $6 C$ & $7 C$ & $7 C^{+}$ \\
60 & $4 S^{+}$ & $6 S^{+}$ & $12 S^{+}$ & $15 S^{+}$ \\
63 & $6 S^{+}$ & $7 S^{+}$ & $8 S^{+}$ & $12 S^{+}$ \\
72 & $4 S^{+}$ & $6 S^{+}$ & $8 C$ & $8 C$ \\
\hline
\end{tabular}

Table 2: Non-self-dual strongly flag-transitive $\left(v_{3}\right)$ configurations, $v \leq 72$. 


\begin{tabular}{|c|c|c|c|c|c|c|c|c|c|}
\hline$v$ & \multicolumn{9}{|c|}{ Weakly rotary orbits } \\
\hline ) & $8 C$ & $8 C$ & $6 S^{+}$ & $10 S^{+}$ & $12 S^{+}$ & $12 S^{+}$ & $12 S^{+}$ & $20 S^{+}$ & $20 S^{+}$ \\
\hline 30 & $6 C$ & $6 C$ & $8 C$ & $8 C$ & $10 C$ & $10 C$ & $8 S^{+}$ & $10 S^{+}$ & $2 S^{+}$ \\
\hline 36 & $8 C$ & $8 C$ & $8 C$ & $8 C$ & $8 C$ & $8 C$ & $8 C$ & $8 C$ & $12 S^{+}$ \\
\hline 36 & $8 C$ & $8 C$ & $16 C$ & $16 C$ & $16 C$ & $16 C$ & $6 S^{+}$ & $12 S^{+}$ & $12 S$ \\
\hline 40 & $S^{+}$ & $8 S^{+}$ & $8 S^{+}$ & $8 S^{+}$ & $10 S^{+}$ & $10 S^{+}$ & $12 S^{+}$ & $12 S^{+}$ & $16 S$ \\
\hline 42 & $4 C$ & $14 C$ & $6 S^{+}$ & $6 S^{+}$ & $8 S^{+}$ & $8 S^{+}$ & $12 S^{+}$ & $12 S^{+}$ & $12 S^{+}$ \\
\hline 43 & $S^{+}$ & $10 S^{+}$ & $12 S^{+}$ & $12 S^{+}$ & $16 S^{+}$ & $18 S^{+}$ & $20 S^{+}$ & $24 S^{+}$ & $24 S^{-}$ \\
\hline 48 & $6 C$ & $6 C$ & $8 C$ & $8 C$ & $12 C$ & $12 C$ & $8 S^{+}$ & $8 S^{+}$ & $8 S^{+}$ \\
\hline 48 & $6 S^{+}$ & $8 S^{+}$ & $8 S^{+}$ & $8 S^{+}$ & $8 S^{+}$ & $8 S^{+}$ & $12 S^{+}$ & $12 S^{+}$ & $12 S^{+}$ \\
\hline 48 & $6 C$ & $6 C$ & $8 C$ & $8 C$ & $8 C$ & $8 C$ & $12 C$ & $12 C$ & $8 S^{+}$ \\
\hline 48 & $12 C$ & $12 C$ & $8 S^{+}$ & $8 S^{+}$ & $8 S^{+}$ & $12 S^{+}$ & $12 S^{+}$ & $16 S^{+}$ & $16 S^{+}$ \\
\hline 48 & $4 C$ & $24 C$ & $8 S^{+}$ & $8 S^{+}$ & $8 S^{+}$ & $16 S^{+}$ & $16 S^{+}$ & $24 S^{+}$ & $24 S^{-}$ \\
\hline 50 & $8 C$ & $8 C$ & $8 C$ & $8 C$ & $20 C$ & $20 C$ & $8 S^{+}$ & $10 S^{+}$ & $20 S^{+}$ \\
\hline 55 & $6 S^{+}$ & $10 S^{+}$ & $10 S^{+}$ & $10 S^{+}$ & $20 S^{+}$ & $20 S^{+}$ & $22 S^{+}$ & $24 S^{+}$ & $24 S^{+}$ \\
\hline 60 & $6 S^{+}$ & $10 S^{+}$ & $12 S^{+}$ & $12 S^{+}$ & $20 S^{+}$ & $20 S^{+}$ & $20 S^{+}$ & $20 S^{+}$ & $20 S^{+}$ \\
\hline 60 & $8 C$ & $8 C$ & $30 C$ & $30 C$ & $12 S^{+}$ & $12 S^{+}$ & $12 S^{+}$ & $20 S^{+}$ & $24 S^{+}$ \\
\hline 60 & $8 C$ & $8 C$ & $10 S^{+}$ & $12 S^{+}$ & $12 S^{+}$ & $12 S^{+}$ & $20 S^{+}$ & $20 S^{+}$ & $24 S^{+}$ \\
\hline 60 & $8 C$ & $8 C$ & $10 C$ & $10 C$ & $12 C$ & $12 C$ & $8 S^{+}$ & $12 S^{+}$ & $20 S^{+}$ \\
\hline 60 & $8 C$ & $8 C$ & $12 C$ & $12 C$ & $20 C$ & $20 C$ & $8 S^{+}$ & $10 S^{+}$ & $12 S^{-}$ \\
\hline 60 & $6 C$ & $6 C$ & $8 C$ & $8 C$ & $20 C$ & $20 C$ & $8 S^{+}$ & $12 S^{+}$ & $20 S^{+}$ \\
\hline 60 & $8 C$ & $8 C$ & $8 C$ & $8 C$ & $12 C$ & $12 C$ & $10 S^{+}$ & $12 S^{+}$ & $20 S^{+}$ \\
\hline 60 & $8 C$ & $8 C$ & $8 C$ & $8 C$ & $24 C$ & $24 C$ & $6 S^{+}$ & $10 S^{+}$ & $20 S^{+}$ \\
\hline 64 & $2 C$ & $32 C$ & $8 S^{+}$ & $8 S^{+}$ & & $16 S^{+}$ & & $16 S^{+}$ & $16 S^{+}$ \\
\hline 64 & $S^{+}$ & $8 S^{+}$ & $8 S^{+}$ & $8 S^{+}$ & $16 S^{+}$ & $16 S^{+}$ & $16 S^{+}$ & $16 S^{+}$ & $16 S^{-}$ \\
\hline 64 & $8 S^{+}$ & $8 S^{+}$ & $8 S^{+}$ & $12 S^{+}$ & $12 S^{+}$ & $16 S^{+}$ & $16 S^{+}$ & $16 S^{+}$ & $24 S^{+}$ \\
\hline 64 & $2 C$ & $32 C$ & $8 S^{+}$ & $8 S^{+}$ & $8 S^{+}$ & $16 S^{+}$ & $16 S^{+}$ & $32 S^{+}$ & $32 S^{+}$ \\
\hline 64 & $S^{+}$ & $8 S^{+}$ & $8 S^{+}$ & $16 S^{+}$ & $16 S^{+}$ & $32 S^{+}$ & $32 S^{+}$ & $32 S^{+}$ & $32 S^{+}$ \\
\hline 64 & $8 S^{+}$ & $8 S^{+}$ & $8 S^{+}$ & $8 S^{+}$ & $8 S^{+}$ & $16 S^{+}$ & $16 S^{+}$ & $16 S^{+}$ & $16 S^{+}$ \\
\hline 64 & $2 C$ & $12 C$ & $12 C$ & $12 C$ & $24 C$ & $24 C$ & $8 S^{+}$ & $16 S^{+}$ & $16 S^{+}$ \\
\hline 64 & $8 S^{+}$ & $8 S^{+}$ & $16 S^{+}$ & $16 S^{+}$ & $16 S^{+}$ & $16 S^{+}$ & $16 S^{+}$ & $16 S^{+}$ & $16 S^{+}$ \\
\hline 72 & $8 C$ & $8 C$ & $16 C$ & $16 C$ & $16 C$ & $16 C$ & $12 S^{+}$ & $12 S^{+}$ & $12 S^{+}$ \\
\hline 72 & $8 C$ & $8 C$ & $8 C$ & $8 C$ & $24 C$ & $24 C$ & $8 S^{+}$ & $12 S^{+}$ & $24 S^{+}$ \\
\hline 72 & $8 S^{+}$ & $8 S^{+}$ & $8 S^{+}$ & $8 S^{+}$ & $8 S^{+}$ & $12 S^{+}$ & $12 S^{+}$ & $12 S^{+}$ & $24 S^{+}$ \\
\hline 72 & $8 C$ & $8 C$ & $16 C$ & $16 C$ & $16 C$ & $16 C$ & $12 S^{+}$ & $12 S^{+}$ & $12 S^{+}$ \\
\hline 72 & $8 C$ & $8 C$ & $16 C$ & $16 C$ & $16 C$ & $16 C$ & $6 S^{+}$ & $12 S^{+}$ & $12 S^{+}$ \\
\hline 72 & $8 C$ & $8 C$ & $12 C$ & $12 C$ & $24 C$ & $24 C$ & $12 S^{+}$ & $12 S^{+}$ & $12 S^{+}$ \\
\hline 72 & $6 S^{+}$ & $8 S^{+}$ & $8 S^{+}$ & $8 S^{+}$ & $8 S^{+}$ & $12 S^{+}$ & $12 S^{+}$ & $12 S^{+}$ & $16 S^{+}$ \\
\hline 72 & $8 S^{+}$ & $8 S^{+}$ & $8 S^{+}$ & $8 S^{+}$ & $12 S^{+}$ & $12 S^{+}$ & $12 S^{+}$ & $16 S^{+}$ & $24 S^{+}$ \\
\hline 75 & $6 C$ & $6 C$ & $8 C$ & $8 C$ & $8 C$ & $8 C$ & $12 C$ & $12 C$ & $10 S^{+}$ \\
\hline
\end{tabular}

Table 3: Non-self-dual strongly flag-transitive $\left(v_{4}\right)$ configurations, $v \leq 75$. 


\begin{tabular}{|c|c|c|c|c|c|c|c|c|c|c|c|c|}
\hline$v$ & \multicolumn{2}{|c|}{ S. rotary } & \multicolumn{3}{|c|}{ W. rotary } & $v$ & \multicolumn{3}{|c|}{ S. rotary } & \multicolumn{3}{|c|}{ W. rotary } \\
\hline 13 & $S^{+} 4 S^{+}$ & $6 S^{+}$ & $8 S^{-}$ & $13 S^{-}$ & $13 S^{-}$ & 39 & $6 C$ & $6 C$ & $13 S^{+}$ & $4 C$ & $4 C$ & $39 S^{-}$ \\
\hline 14 & $3 S^{+} 4 S^{+}$ & $4 S^{+}$ & $7 S^{-}$ & $14 S^{-}$ & $6 S^{+}$ & 40 & $8 C$ & $8 C$ & $5 S^{+}$ & $4 C$ & $4 C$ & $40 S^{-}$ \\
\hline 15 & $3 S^{+} \quad 5 S^{+}$ & $6 S^{+}$ & $4 S^{-}$ & $15 S^{-}$ & $6 S^{+}$ & 40 & $4 C$ & $4 C$ & $10 S^{+}$ & $4 C$ & $4 C$ & $20 S^{-}$ \\
\hline 16 & $3 S^{+} \quad 4 S^{+}$ & $6 S^{+}$ & $8 S^{-}$ & $4 S^{+}$ & $6 S^{+}$ & 40 & $8 C$ & $8 C$ & $10 S^{+}$ & $4 C$ & $4 C$ & $40 S^{-}$ \\
\hline 18 & $4 C \quad 4 C$ & $3 S^{+}$ & $4 C$ & $4 C$ & $4 S^{-}$ & 40 & $4 C$ & $4 C$ & $10 S^{+}$ & $4 C$ & $4 C$ & $10 S^{-}$ \\
\hline 20 & $4 C \quad 4 C$ & $10 S^{+}$ & $4 C$ & $4 C$ & $20 S^{-}$ & 42 & $3 S^{+}$ & $4 S^{+}$ & $6 S^{+}$ & $6 S^{+}$ & $8 S^{+}$ & $14 S^{+}$ \\
\hline 20 & $4 C \quad 4 C$ & $5 S^{+}$ & $4 C$ & $4 C$ & $20 S^{-}$ & 42 & $3 S^{+}$ & $4 S^{+}$ & $7 S^{+}$ & $8 S^{-}$ & $8 S^{+}$ & $7 S^{+}$ \\
\hline 21 & $3 S^{+} 3 S^{+}$ & $4 S^{+}$ & $6 S^{+}$ & $7 S^{+}$ & $8 S^{+}$ & 42 & $3 S^{+}$ & $4 S^{+}$ & $12 S^{+}$ & $14 S^{-}$ & $21 S^{-}$ & $6 S^{+}$ \\
\hline 24 & $4 S^{+} \quad 6 S^{+}$ & $12 S^{+}$ & $12 S^{-}$ & $8 S^{+}$ & $8 S^{+}$ & 42 & $3 S^{+}$ & $4 S^{+}$ & $4 S^{+}$ & $14 S^{-}$ & $3 S^{+}$ & $6 S^{+}$ \\
\hline 24 & $3 S^{+} \quad 4 S^{+}$ & $12 S^{+}$ & $12 S^{-}$ & $8 S^{+}$ & $8 S^{+}$ & 45 & $8 C$ & $18 C$ & $5 S^{+}$ & $4 C$ & $4 C$ & $45 S^{-}$ \\
\hline 24 & $3 S^{+} \quad 4 S^{+}$ & $6 S^{+}$ & $8 S^{+}$ & $8 S^{+}$ & $12 S^{+}$ & 45 & $3 S^{+}$ & $10 S^{+}$ & $15 S^{+}$ & $30 S^{-}$ & $4 S^{+}$ & $6 S^{+}$ \\
\hline 25 & $10 C 10 C$ & $5 S^{+}$ & $4 C$ & $4 C$ & $5 S^{-}$ & 45 & $3 S^{+}$ & $4 S^{+}$ & $5 S^{+}$ & $6 S^{-}$ & $8 S^{+}$ & $10 S^{+}$ \\
\hline 27 & $3 S^{+} 3 S^{+}$ & $6 S^{+}$ & $6 S^{-}$ & $6 S^{+}$ & $12 S^{+}$ & 48 & $6 S^{+}$ & $8 S^{+}$ & $24 S^{+}$ & $4 S^{+}$ & $8 S^{+}$ & $12 S^{+}$ \\
\hline 27 & $3 S^{+} 3 S^{+}$ & $6 S^{+}$ & $6 S^{-}$ & $9 S^{-}$ & $4 S^{+}$ & 48 & $8 S^{+}$ & $12 S^{+}$ & $24 S^{+}$ & $4 S^{+}$ & $6 S^{+}$ & $8 S^{+}$ \\
\hline 30 & $6 C \quad 6 C$ & $5 S^{+}$ & $4 C$ & $4 C$ & $30 S^{-}$ & 48 & $4 S^{+}$ & $24 S^{+}$ & $24 S^{+}$ & $8 S^{+}$ & $8 S^{+}$ & $24 S^{+}$ \\
\hline 30 & $3 S^{+} 3 S^{+}$ & $5 S^{+}$ & $4 S^{-}$ & $6 S^{+}$ & $10 S^{+}$ & 48 & $4 S^{+}$ & $6 S^{+}$ & $12 S^{+}$ & $24 S^{-}$ & $4 S^{+}$ & $4 S^{+}$ \\
\hline 30 & $3 S^{+} \quad 5 S^{+}$ & $5 S^{+}$ & $5 S^{+}$ & $6 S^{+}$ & $10 S^{+}$ & 48 & $4 S^{+}$ & $12 S^{+}$ & $12 S^{+}$ & $24 S^{-}$ & $4 S^{+}$ & $4 S^{+}$ \\
\hline 32 & $4 S^{+} 8 S^{+}$ & $8 S^{+}$ & $4 S^{+}$ & $4 S^{+}$ & $8 S^{+}$ & 48 & $3 S^{+}$ & $4 S^{+}$ & $6 S^{+}$ & $8 S^{+}$ & $8 S^{+}$ & $12 S^{+}$ \\
\hline 32 & $4 S^{+} \quad 4 S^{+}$ & $8 S^{+}$ & $8 S^{-}$ & $4 S^{+}$ & $8 S^{+}$ & 48 & $3 S^{+}$ & $3 S^{+}$ & $4 S^{+}$ & $8 S^{+}$ & $8 S^{+}$ & $6 S^{+}$ \\
\hline 32 & $8 C \quad 8 C$ & $4 S^{+}$ & $4 C$ & $4 C$ & $4 S^{-}$ & 48 & $4 S^{+}$ & $6 S^{+}$ & $12 S^{+}$ & $4 S^{+}$ & $4 S^{+}$ & $12 S^{+}$ \\
\hline 32 & $3 S^{+} \quad 4 S^{+}$ & $4 S^{+}$ & $6 S^{-}$ & $8 S^{-}$ & $4 S^{+}$ & 48 & $4 S^{+}$ & $6 S^{+}$ & $6 S^{+}$ & $8 S^{+}$ & $8 S^{+}$ & $12 S^{+}$ \\
\hline 32 & $4 S^{+} 8 S^{+}$ & $8 S^{+}$ & $4 S^{-}$ & $4 S^{+}$ & $4 S^{+}$ & 48 & $4 S^{+}$ & $6 S^{+}$ & $12 S^{+}$ & $12 S^{-}$ & $8 S^{+}$ & $8 S^{+}$ \\
\hline 35 & $14 C 14 C$ & $5 S^{+}$ & $4 C$ & $4 C$ & $35 S^{-}$ & 48 & $4 S^{+}$ & $6 S^{+}$ & $12 S^{+}$ & $6 S^{-}$ & $8 S^{+}$ & $8 S^{+}$ \\
\hline 35 & $3 S^{+} \quad 5 S^{+}$ & $7 S^{+}$ & $4 S^{+}$ & $5 S^{+}$ & $6 S^{+}$ & 48 & $4 S^{+}$ & $6 S^{+}$ & $12 S^{+}$ & $4 S^{+}$ & $4 S^{+}$ & $6 S^{+}$ \\
\hline 36 & $4 S^{+} \quad 6 S^{+}$ & $12 S^{+}$ & $6 S^{-}$ & $4 S^{+}$ & $6 S^{+}$ & 48 & $3 S^{+}$ & $4 S^{+}$ & $12 S^{+}$ & $4 S^{+}$ & $4 S^{+}$ & $6 S^{+}$ \\
\hline 36 & $3 S^{+} \quad 4 S^{+}$ & $12 S^{+}$ & $12 S^{-}$ & $4 S^{+}$ & $6 S^{+}$ & 48 & $3 S^{+}$ & $4 S^{+}$ & $6 S^{+}$ & $12 S^{-}$ & $4 S^{+}$ & $4 S^{+}$ \\
\hline 36 & $3 S^{+} 3 S^{+}$ & $6 S^{+}$ & $4 S^{+}$ & $6 S^{+}$ & $12 S^{+}$ & 48 & $3 C$ & $3 C$ & $4 S^{+}$ & $4 C$ & $4 C$ & $6 S^{-}$ \\
\hline 36 & $4 C \quad 4 C$ & $3 S^{+}$ & $4 C$ & $4 C$ & $4 S^{-}$ & & & & & & & \\
\hline
\end{tabular}

Table 4: Self-dual strongly flag-transitive $\left(v_{4}\right)$ configurations, $v \leq 48$. 


\begin{tabular}{|c|c|c|c|c|c|}
\hline & rotary & Weakly rotary & $v$ & Strongly rotary & kly rotary \\
\hline 27 & $\begin{array}{llll}6 C & 6 C & 18 C & 18 C\end{array}$ & $12 C 12 C 18 S^{+}$ & 57 & \begin{tabular}{|llll}
$6 C$ & $6 C$ & $6 C$ & $6 C$
\end{tabular} & $12 C$ \\
\hline 34 & $8 C \quad 8 C \quad 8 C$ & $8 C$ & 60 & $8 C$ & \\
\hline $3 !$ & $12 C$ & $6 C$ & 60 & $8 C^{\circ} 8 C^{\circ}$ & \\
\hline & $\mathrm{C}$ & $12 C 1$ & 03 & 180 & \\
\hline $42-2$ & $12 C 12 C 12$ & 1 & 63 & $6 C \quad 6 C$ & 12 \\
\hline 4 & $6 C$ & 1 & 64 & $16 C \quad 16 C 32 C 32 C$ & $8 C$ \\
\hline 4 & $12 C 12 C 3$ & $12 C 12 C$ & $64 \mid$ & $8 C \quad 32 C \quad 32 C$ & $16 C 16 C$ \\
\hline & $6 C$ & $2 C 1$ & 68 & $8 C \quad 8 C \quad 8 C \quad 8 C$ & $8 C$ \\
\hline & $12 C 12 C 18$ & C $12 C$ & 68 & $16 C \quad 16 C \quad 16 C \quad 16 C$ & $16 C 16$ \\
\hline & $C 10 C 1 \mathrm{C}$ & $20 C 20 C$ & 68 & $16 C \quad 16 C \quad 16 C \quad 16 C$ & $16 C 16 C 34 S^{-}$ \\
\hline & & & & & \\
\hline
\end{tabular}

Table 5: Self-dual weakly flag-transitive $\left(v_{4}\right)$ configurations, $v \leq 68$. 


\section{References}

[1] N. Biggs, Aspects of symmetry in graphs, Algebraic methods in graph theory, Vol. I, II (Szeged, 1978), pp. 27-35, Colloq. Math. Soc. János Bolyai, 25, North-Holland, Amsterdam-New York, 1981.

[2] M. Boben, B. Grünbaum, T. Pisanski, A. Žitnik, Small triangle-free configurations of points and lines, Discrete Comput. Geom. 35 (2006), 405-427.

[3] M. Boben, Š. Miklavič, P. Potočnik, Consistent cycles in $\frac{1}{2}$-arc-transitive graphs, Electronic J. Comb. 16 (2009), \#R5.

[4] M. D. E. Conder and P. Dobcsányi, Trivalent symmetric graphs on up to 768 vertices, J. Combin. Math. Combin. Comput. 40 (2002), 41-63.

[5] M. C. E. Conder, A. Malnič, D. Marušič, T. Pisanski, P. Potočnik, The cubic edgebut not vertex-transitive graph on 112 vertices, J. Graph Theory 50 (2005), 25-42.

[6] M. C. E. Conder, A. Malnič, D. Marušič, P. Potočnik, A census of cubic semisymmetric graphs on up to 768 vertices, J. Alg. Combin. 23 (2006), 255-294.

[7] B. Grünbaum, J. F. Rigby, The real configuration (214), J. London Math. Soc. 41 (1990), 336-346.

[8] D. Marušič, T. Pisanski, Weakly flag-transitive configurations and half-arc-transitive graphs, Europ. J. Combin. 20 (1999), 559-570.

[9] Š. Miklavič, P. Potočnik, S. Wilson, Overlap in consistent cycles, J. Graph Theory 55 (2007), 55-71.

[10] A. Schönflies, Über die regelmässigen Configurationen $n_{3}$, Math. Ann. 31 (1888), 43-69.

[11] E. Steinitz, Über die Unmöglichkeit, gewisse Configurationen $n_{3}$ in einem geschlossenen Zuge zu durchlaufen, Monatshefte Math. Phys. 8 (1897), 293-296.

[12] S. Wilson, A Census of edge-transitive tetravalent graphs, http://jan.ucc.nau.edu/ swilson/C4Site/index.html. 